\title{
NEURAL MODEL FOR FAR-FIELD 1D LOCALIZATION OF MOBILE STOCHASTIC EM SOURCES WITH PARTIALLY CORRELATED RADIATION
}

\author{
Zoran Stanković, \\ Nebojša Doncov ${ }^{1}$, \\ Ivan Milovanović2, \\ Maja Sarevska ${ }^{3}$, \\ Bratislav Milovanović ${ }^{2}$
}

${ }^{1}$ Faculty of Electronic Engineering, University of Nis, Serbia 2Singidunum University, Belgrade, Serbia

${ }^{3}$ European University - Republic of Macedonia (EURM)

Correspondence:

Zoran Stanković

e-mail:

zoran.stankovic@elfak.ni.ac.rs

\begin{abstract}
:
This paper considers a possibility of angle position determination of mobile stochastic sources with partially correlated radiation, where antenna array and multilayer perceptron neural network processing are used. It is shown that the neural model trained with samples from a system with uncorrelated source radiation cannot determine position of sources with a satisfactory accuracy when sources have some degree of correlation in radiation. That is why it is suggested training samples to be generated for different values of partial source correlation. This kind of generated neural network training may provide source position determination with satisfactory accuracy even when there is partial correlation, which in the paper is presented with an example of two sources that linearly move in azimuth plane
\end{abstract}

Keywords:

Source localization, stochastic radiation, correlation matrix, neural networks.

\section{INTRODUCTION}

Adaptive antenna systems application [1] and techniques based on space signal processing are used as an important strategy for finding a solution of minimizing negative effects of interference on reception side, capacity increasing and service improvement of modern wireless communication systems. Within above mention strategy applications, DoA (Direction of Arrival) estimation and space source location determination are of great importance [1,2].

So far the most common methods are those based on superresolution algorithms like for example MUSIC and its modifications [1,2]. These methods provide a high accuracy in space location estimation of the radiation sources, but because of complex matrix estimations they demand powerful hardware resources and are not convenient for real time applications. Artificial neural networks [3-5] may be applied in the process of DoA estimation of radiation EM sources, as neural models that avoid intensive matrix estimations may be a good alternative to superresolution algorithms. These neural solutions may achieve same accuracy while gaining higher speeds in response. This is shown in [5-11], with a particular emphasis on neural models for 1D DoA estimation [6,7] and neural model for 2D DoA estimation of deterministic radiation sources [8]. 
Today, a high attention is paid for interference sources that have stochastic radiation nature $[12,13]$, so spatial location estimation of this kind of sources is of crucial interest. In [9-11] the neural models for 1D DoA $[9,10]$ and 2D DoA estimation [11] and spatial position estimation of stochastic radiation sources are presented, developed for sources whose radiation is mutually uncorrelated. This paper continues research work of $[9,10]$ with the aim to investigate the possibility of multilayer perceptron neural network (MLP) application (MLP) [3-5] in DoA estimation for stochastic radiation sources for the case when there is some level of partial correlation in their radiations.

\section{STOCHASTIC EM SOURCE RADIATION MODEL}

In this paper, the same model of stochastic EM source radiation in a far-field zone, as in [9-11], is used. Based on this model, a radiation of a number of stochastic EM sources in far-field is described by a radiation of short dipoles. Each dipole fed by current of stochastic nature represents one stochastic source. The movement scenario that is used in this paper may be described by the azimuth plane and sources disposition in one linear direction (1D movement scenario), while the dipole directions are perpendicular to the azimuth plane. If the total number of sources is $\mathrm{S}$, and if fed current of dipoles can be described by the vector $\mathrm{I}=[\mathrm{I} 1, \mathrm{I} 2, \ldots, \mathrm{IS}])$, the mutual correlation of stochastic sources radiation is described by the correlation matrix $[12,13]$ :

$$
\mathbf{c}^{I}(\omega)=\lim _{T \rightarrow \infty} \frac{1}{2 T}\left[\mathbf{I}(\omega) \mathbf{I}(\omega)^{H}\right]
$$

In far-field, the electric field strength at the selected sampling point can be calculated as:

$$
E(\theta, \varphi)=\mathbf{M}(\theta, \varphi) \mathbf{I}
$$

where $\mathrm{M}$ represents the mapping by Green function

$\mathbf{M}(\theta, \varphi)=j z_{0} \frac{F(\theta, \varphi)}{2 \pi}\left[\frac{e^{j k r_{1}}}{r_{1}} \quad \frac{e^{j k r_{2}}}{r_{2}} \ldots \frac{e^{j k r_{s}}}{r_{s}} \ldots \frac{e^{j k r_{S}}}{r_{S}}\right]$ (3)

$\theta$ and $\varphi$ represent the spatial angles of stochastic source location with respect to the selected sampling point in far-field, $F(\theta, \varphi)$ is the radiation pattern of the short dipole, $r_{S}$ is the distance between s-th stochastic EM source and selected sampling point, $\mathrm{z}_{0}$ is the impedance of free space and $k$ is the phase constant $(k=2 \pi / \lambda)$.
In our scenario the sampling of signals is done with uniform linear antenna array so that the sampling points are on the places of the antenna sensors (Fig.1.). The mutual distance of antenna sensors is $y_{d}$, and total number of sensors is $M$. Axis of antenna array is parallel to the direction of radiation source movement. The correlation matrix of signals received in $Y_{1}, Y_{2}, \ldots, Y_{M}$ sampling points can be obtained from the correlation matrix of antenna elements feed currents as

$$
\begin{aligned}
& \tilde{\mathbf{C}}_{E}[i, j]=\mathbf{M}\left(\theta_{i}, \varphi_{i}\right) \mathbf{c}^{I} \mathbf{M}\left(\theta_{j}, \varphi_{j}\right)^{H} \\
& i=1, \ldots, M j=1, \ldots, M
\end{aligned}
$$

Because in our scenario the sources are moving in azimuth plane, it is assumed that $\varphi=0$ for any angular position of the source. In that case the angle position of $s$-th source in relation to $m$-th antenna array element is

$$
\theta_{s}^{(m)}=\arctan \left[\tan \theta_{s}^{(1)}-\frac{(m-1) \cdot y_{d}}{r_{0}}\right]
$$

while the distance of $s$-th source in relation to the $m$-th antenna array element is

$$
r_{s}^{(m)}=\frac{r_{0}}{\cos \theta_{s}^{(m)}}
$$

Under angle position of $s$-th source in relation to antenna array $\theta_{\mathrm{s}}$ we mean its angle position in relation to the first element of the antenna array when $\theta_{S}=\theta_{s}^{(1)}$. Using (3), (5) and (6) for given angle position of radiation source we may determine the mapping function $\mathbf{M}$, and afterwards also the elements of the correlation matrix using (4). In this paper we use moving of two partially correlated sources with stochastic nature $(S=2)$ that is illustrated in Fig.1. If the correlation degree of their radiation is $c$ then their correlation matrix is

$$
\mathbf{c}^{I}=\left[\begin{array}{cc}
1 & c_{12}^{I} \\
c_{21}^{I} & 1
\end{array}\right]
$$

where $c=c_{12}{ }^{\mathrm{I}}=c_{21}{ }^{\mathrm{I}}$. For neural model training we use the correlation matrix that is normalized with the first matrix element 


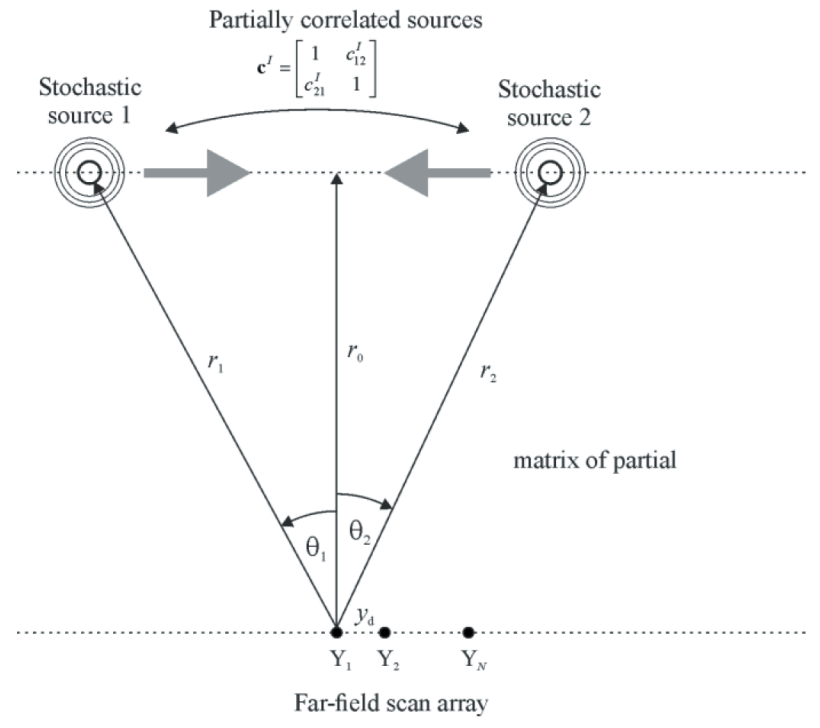

Fig. 1. Architecture of MLP neural model for DoA estimation of two stochastic EM source signal in azimuth plane

$$
\mathbf{C}_{E}=\frac{1}{\tilde{C}_{E 11}} \cdot \tilde{\mathbf{C}}_{E}
$$

\section{NEURAL NETWORK MODEL}

For neural network model realization the same model architecture as in [9], is used. The neural model is based on MLP ANN [3-5], and for the case which considers the two stochastic sources, its architecture is shown in Fig.2. The main purpose of the model is to perform the mapping from the space of $S$ signals described by correlation matrix $\mathrm{C}_{\mathrm{E}}$ to the $1 \mathrm{D}$ DoA space

$$
\left[\theta_{1} \theta_{2}\right]^{T}=f\left(\mathbf{C}_{E}\right)
$$

where $\left[\begin{array}{ll}\theta 1 & \theta 2\end{array}\right]^{\mathrm{T}}$ is vector of azimuth angles of arrival of the stochastic sources radiation. Its MLP network can be described by the following function:

$$
\mathbf{y}_{l}=F\left(\mathbf{w}_{l} \mathbf{y}_{l-1}+\mathbf{b}_{l}\right) \quad l=1,2, \ldots, H
$$

where $y_{1}-1$ vector represents the output of (l-1)-th hidden layer, wl is a connection weight matrix among (l-1)-th and $l$-th hidden layer neurons, $b_{1}$ is a vector containing biases of $l$-th hidden layer neurons and $H$ is number of hidden layers. $F$ is the activation function of neurons in hidden layers and in this case it is a hyperbolic tangent sigmoid transfer function:

$$
F(u)=\frac{e^{u}-e^{-u}}{e^{u}+e^{-u}}
$$

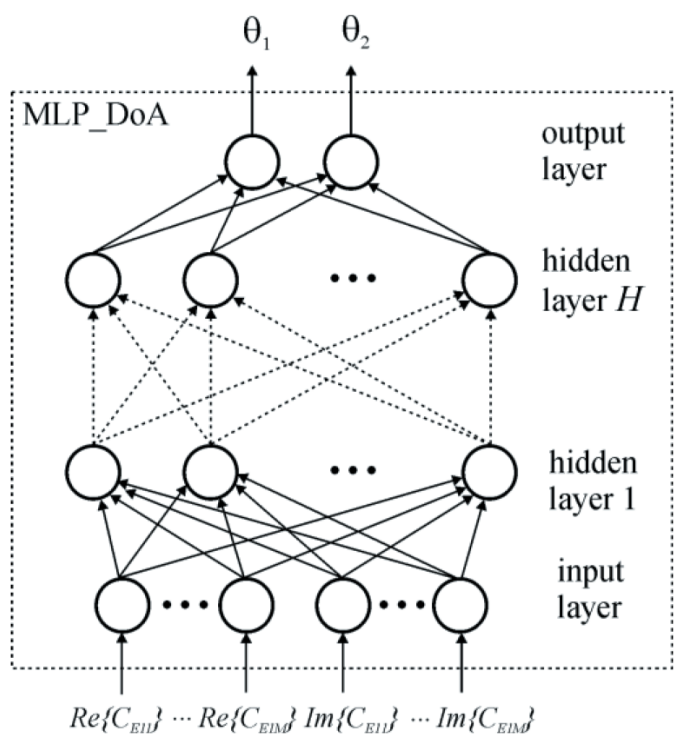

Fig. 2. Architecture of MLP neural model for DoA estimation of two stochastic EM source signal in azimuth plane (MLP_DoA model)

In order to perform mapping it is sufficient to take only the first row of correlation matrix and accordingly the input layer receives data in the format $\mathrm{x}=\mathrm{y} 0=\left[\operatorname{Re}\left\{C_{E}[1,1]\right\}, \ldots\right.$, $\left.\operatorname{Re}\left\{C_{E}[1, M]\right\}, \operatorname{Im}\left\{C_{E}[1,1]\right\}, \ldots, \operatorname{Im}\left\{C_{E}[1, M]\right\}\right]^{\mathrm{T}}$. Also, the output of the neural network model is given as

$\left[\begin{array}{ll}\theta 1 & \theta 2\end{array}\right]^{\mathrm{T}}=\mathrm{w}_{\mathrm{H}}+1 \mathrm{y}_{\mathrm{H}}$ where $\mathrm{w}_{\mathrm{H}}+1$ is a connection weight matrix between neurons of last hidden layer and neurons in the output layer. The optimization of weight matrices $\mathrm{w}_{1}, \mathrm{w}_{2}, \ldots, \mathrm{w}_{\mathrm{H}}, \mathrm{w}_{\mathrm{H}}+1$ and biases values during the training allows ANN to approximate the mapping with the desired accuracy.

The general designation for this defined MLP neural model is MLPH-N $\mathrm{N}_{1}-\ldots-\mathrm{Ni}-\ldots-\mathrm{N}_{\mathrm{H}}$ where $\mathrm{H}$ is the total number of hidden layers used in MLP network, while $\mathrm{Ni}$ is the total number of neurons in the $i$-th hidden layer.

\section{MODELING RESULTS}

MLP_DoA model, whose architecture was presented in previous section, is applied for DoA estimation of two stochastic sources that independently from each other move along linear trajectory distant $r_{0}=100 \mathrm{~m}$ from the antenna array, with whom the signal is sampled at frequency $f=28 \mathrm{GHz}$. The scanning width of antenna array in the azimuth is $\left[-30^{\circ} 30^{\circ}\right]$. We analyze two cases. 
In the first case we assume that the sources are mutually uncorrelated, and in the second, we assume that there is mutual partial correlation in the range $0.05 \leq \mathrm{c} \leq 0.8$. For both cases we generated separate sample sets for network training and testing, while using common parameters of sampling that are given in table I.

For training and testing samples generation we use relations (3) and (4) that establish inverse mapping from that of the MLP_DoA model

$$
\mathbf{C}_{E}^{t}=f_{D o A}^{-1}\left(\theta_{1}^{t}, \theta_{1}^{t}, c\right)
$$

So the samples for neural network training and testing are given in the format $\left\{\left(x^{\mathrm{t}}\left(\theta_{1}^{\mathrm{t}}, \theta_{2}^{\mathrm{t}}, c^{\mathrm{t}}\right), \theta_{1}^{\mathrm{t}}, \theta_{2}^{\mathrm{t}}\right)\right\}$, where $x^{t}$ is vector of input model values $x^{t}=\left[\operatorname{Re}\left\{C_{E}^{t}[1,1]\right\}, \ldots\right.$, $\left.\operatorname{Re}\left\{C_{E}^{t}[1, \mathrm{M}]\right\}, \operatorname{Im}\left\{C_{E}^{t}[1,1]\right\}, \ldots, \operatorname{Im}\left\{C_{E}^{t}[1, M]\right\}\right]^{\mathrm{T}}$. For each element of vector xt we used uniform distribution of samples for azimuth angles of radiation source location and correlation of the form [ $\theta^{t} \min : \theta^{t}$ step : $\left.\theta^{t} \max \right]$ and [ $c^{t} \min : c^{t}$ step : $\left.c^{t} \max \right]$, where $\theta^{t} \min \left[{ }^{\circ}\right]$ and $c^{t} \min$ represent the lowest limit of distribution, $\theta^{t} \max \left[{ }^{\circ}\right]$ and $c^{t} \max$ represent the highest limit of distribution, while $\theta^{\text {tstep }}$ and $c^{t}$ step represent uniform sampling steps.

\begin{tabular}{lc}
\hline Frequency & $f=28 \mathrm{GHz}$ \\
\hline Number of sources & $S=2$ \\
\hline Sampling points distance from linear source trajectory & $r_{0}=100 \mathrm{~m}$ \\
\hline Number of sampling antenna array sensors & $M=6$ \\
\hline Mutual distance of the antenna sensors & $s=\lambda / 2(5.4 \mathrm{~mm})$ \\
\hline
\end{tabular}

Table 1. Sampling parameters which are used for training and test sets generating

For model training and testing in the first case when we assume uncorrelated stochastic sources the following sets are generated:

TRAINING_A set (28920 samples):

$\left\{\begin{array}{l}\left(\mathbf{x}^{t}\left(\theta_{1}^{t}, \theta_{2}^{t}, 0\right), \theta_{1}^{t}, \theta_{2}^{t}\right) \mid \\ \theta_{1}^{t} \in[-30: 0.25: 30], \theta_{2}^{t} \in[-30: 0.25: 30], \theta_{1}^{t}>\theta_{2}^{t}\end{array}\right\}$

TEST_A set (5050 samples):

$\left\{\begin{array}{l}\left(\mathbf{x}^{t}\left(\theta_{1}^{t}, \theta_{2}^{t}, 0\right), \theta_{1}^{t}, \theta_{2}^{t}\right) \mid \\ \theta_{1}^{t} \in[-30: 0.6: 30], \theta_{2}^{t} \in[-30: 0.6: 30], \theta_{1}^{t}>\theta_{2}^{t}\end{array}\right\}$

For model training and testing in the second case when we assume that the stochastic sources may have some degree of correlation $c$, following sets are generated:
TRAINING_B set (29280 samples):

$$
\left\{\begin{array}{l}
\left(\mathbf{x}^{t}\left(\theta_{1}^{t}, \theta_{2}^{t}, c^{t}\right), \theta_{1}^{t}, \theta_{2}^{t}\right) \mid \\
\theta_{1}^{t} \in[-30: 1: 30], \theta_{2}^{t} \in[-30: 1: 30], \theta_{1}^{t}>\theta_{2}^{t} \\
c^{t} \in[0.05: 0.05: 0.8]
\end{array}\right\}
$$

TEST_B set (3036 samples):

$\left\{\begin{array}{l}\left(\mathbf{x}^{t}\left(\theta_{1}^{t}, \theta_{2}^{t}, c^{t}\right), \theta_{1}^{t}, \theta_{2}^{t}\right) \mid \\ \theta_{1}^{t} \in[-30: 2.7: 30], \theta_{2}^{t} \in[-30: 2.7: 30], \theta_{1}^{t}>\theta_{2}^{t} \\ c^{t} \in[0: 0.07: 0.8]\end{array}\right\}$

In both cases the model was realized using a network with two hidden layers $(\mathrm{H}=2)$, and in order to get a model with higher accuracy the training was done for a number of MLP networks, with different number of neurons in the hidden layers (MLP2-N1-N2, $4 \leq N 1, N 2 \leq 23$ ). In the process of testing we analyzed the values of worst case error (WCE) and average error (ACE) $[4,5]$ that model was showing on testing samples.

In the first case the training of MLP neural model network is done with the set TRAINING_A, on samples that are generated under conditions of uncorrelated radiation of the stochastic sources. Afterwards the trained neural networks are tested with the set TEST_A that contains samples that are generated also when the sources are uncorrelated. The results of the testing of the six models with lowest value of WCE on the set TEST_A are shown in Table II. Figures 3.(a) i 3.(b) present scattering diagram of MLP2-12-7 model on TEST_A set (this model has shown lowest WCE on that set). It can be seen that all six models show high accuracy in source location estimation. But if in the network input we deliver samples that are generated with some source radiation correlation (set TEST_B) then the models show high WCE value or high imprecision in source location estimation. That may be seen also in Table II also from scattering diagram of MLP2-12-7 model on TEST_B set (Fig 4.(a) and 4.(b)).

\begin{tabular}{ccccc}
\hline \multirow{2}{*}{ MLP model } & \multicolumn{2}{c}{ TEST_A set } & \multicolumn{2}{c}{ TEST_B set } \\
\cline { 2 - 5 } & WCE [\%] & ACE [\%] & WCE [\%] & ACE [\%] \\
\hline MLP2-12-7 & 2.25 & 0.36 & 60.63 & 6.76 \\
\hline MLP2-13-13 & 2.26 & 0.38 & 87.81 & 6.01 \\
\hline MLP2-11-4 & 2.38 & 0.37 & 87.31 & 7.16 \\
\hline MLP2-16-16 & 2.43 & 0.37 & 59.02 & 6.07 \\
\hline MLP2-12-12 & 2.51 & 0.37 & 89.37 & 7.17 \\
\hline MLP2-12-5 & 2.55 & 0.37 & 83.99 & 6.91 \\
\hline
\end{tabular}

Table 2. Testing results for six MLP neural models trained on

TRAINING_A set with the best average errors statistics 
In the second case MLP neural model training is performed on the set TRAINING_B, on samples that are generated under conditions when there is some correlation degree between radiation sources (partial radiation correlation is in the range $0.05 \leq c \leq 0.8$ ). Afterwards the trained neuron networks are tested with the set TEST_B that contains samples that are generated also under variable radiation correlation in above range but also with samples that are generated under no correlation, which is important to be mentioned for this case. The results from testing of the six models with lowest value for WCE on set TEST_B are shown in Table III. On figures 5.(a) and 5.(b) we may see scattering diagram of the MLP2-22-22 model on the set TEST_B (this model has shown lowest value of WCE on that set). It can be seen that the model MLP2-22-22 has satisfactory accuracy in angle position determination when we use samples for different partial correlations of radiating sources.

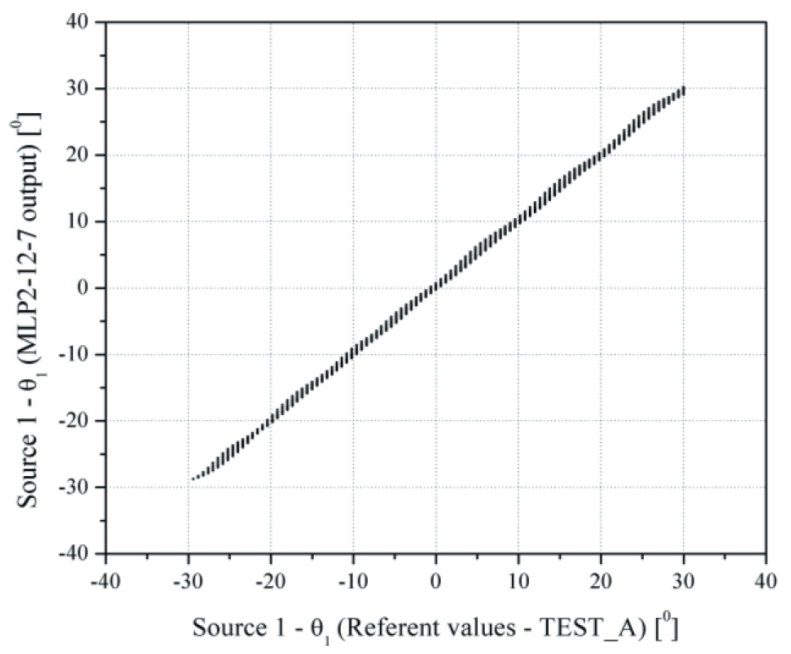

(a)

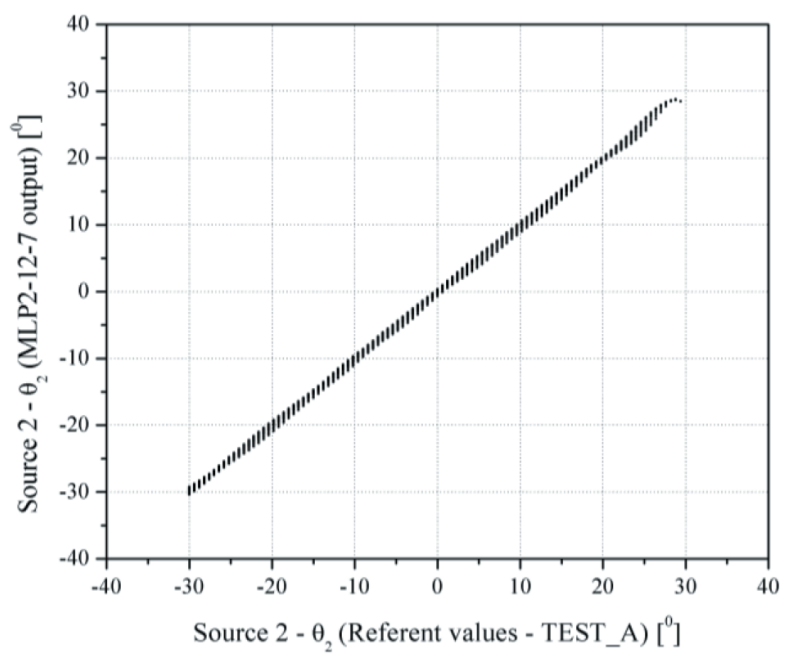

(b)

Fig. 3. Scattering diagram of MLP2-12-7 model $\theta_{1}$ output (a), and $\theta_{2}$ output (b), on TEST_A set

\begin{tabular}{lcc}
\hline & \multicolumn{3}{c}{ TEST_B set } \\
\cline { 2 - 3 } MLP model & WCE [\%] & ACE [\%] \\
\hline MLP2-22-22 & 4.53 & 0.35 \\
\hline MLP2-23-23 & 5.58 & 0.33 \\
\hline MLP2-22-20 & 5.70 & 0.36 \\
\hline MLP2-18-16 & 5.74 & 0.36 \\
\hline MLP2-18-18 & 6.33 & 0.34 \\
\hline MLP2-16-16 & 8.27 & 0.35 \\
\hline
\end{tabular}

Table 3. Testing results for six MLP neural models trained on TRAINING_B set with the best average errors statistics

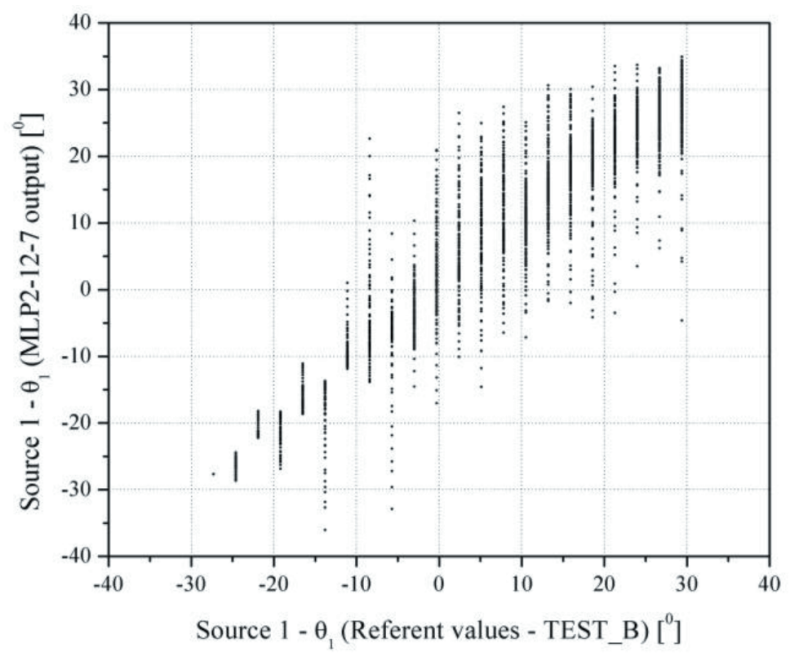

(a)

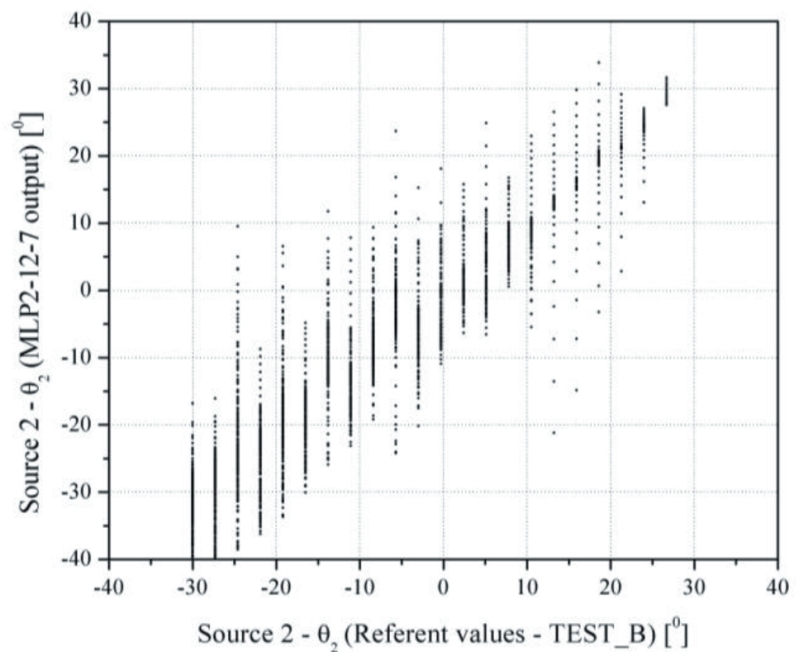

(b)

Fig. 4. Scattering diagram of MLP2-12-7 model $\theta_{1}$ output (a), and $\theta_{2}$ output (b), on TEST_B set 


\section{CONCLUSIONS}

In the simple scenarios (1D) for stochastic sources movements neural models based on MLP networks may be faster and less hardware demanding alternative compared to classic superresolution algorithms for the methods for space localization of stochastic sources. It is shown that the neural models that are trained with samples generated under conditions when the sources have no correlation cannot be used for space source location determination when the sources have correlated radiation. In order neural model to be used with satisfactory accuracy also for correlated sources, that information must be included in training set also, that means that the training set must be generated under conditions when there is different degrees of mutual correlation between the radiating sources. It is shown that that kind of trained model may be used for space source location determination, with satisfactory accuracy, also when the sources are uncorrelated.

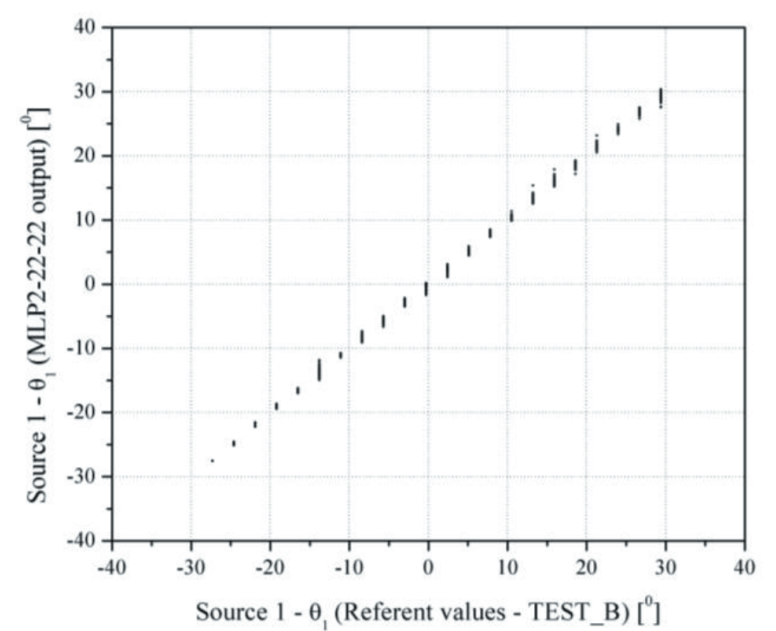

(a)

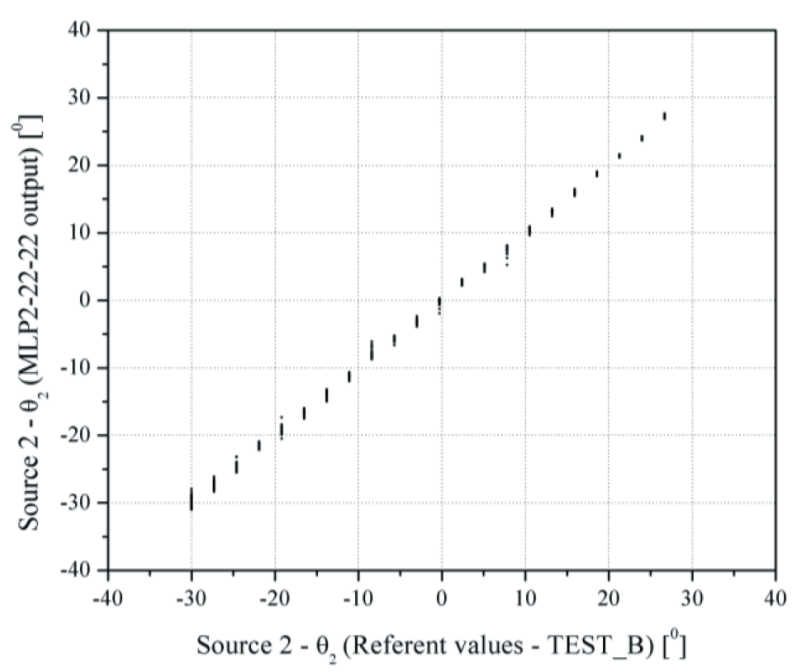

(b)

Fig. 5. Scattering diagram of MLP2-22-22 model $\theta_{1}$ output (a), and $\theta_{2}$ output (b), on TEST_B set

\section{ACKNOWLEDGMENT}

This work has been supported by the Ministry for Education, Science and Technological Development of Serbia, project number TR32052.

\section{REFERENCES}

[1] B. Allen, M. Ghavami, Adaptive Array Systems: fundamentals and applications, Wiley, 2005

[2] R. Schmidt, "Multiple emitter location and signal parameter estimation", IEEE Transactions on Antennas and Propagation, vol. 34, no. 3, pp. 276-28, 1986.

[3] S. Haykin, Neural Networks, New York, IEEE, 1994.

[4] Q. J. Zhang, K. C. Gupta, Neural networks for RF and microwave design, Artech House, Boston, MA, 2000.

[5] C. G. Christodoulou, M. Georgiopoulos, Application of neural networks in electromagnetics, Artech House, December 2000.

[6] A. H. El Zooghby, C. G. Christodoulou, M. Georgiopoulos, "A neural network based smart antenna for multiple source tracking", IEEE Transactions on Antennas and Propagation, Vol. 48, no. 5, pp. 768 776, 2000.

[7] Maja Sarevska, Bratislav Milovanović, Zoran Stanković, "Neural Network - Based DOA Estimation and Beamforming for Smart Antenna", ICEST 2006 Conference Proceedings, Sofija, Bugarska, 29. Jun- 1. Jul 2006, pp. 25-28.

[8] M. Agatonović, Z. Stanković, N. Dončov, L. Sit, B. Milovanović, T. Zwick, "Application of artificial neural networks for efficient high-resolution 2D DOA estimation", Radioengineering, Vol. 21, No. 4, pp. 1178-1186, 2012.

[9] Z. Stankovic, N. Doncov, I. Milovanovic, B. Milovanovic, M. Stoiljkovic, "Localization of mobile users of stochastic radiation nature by using Neural Networks", Proceedings of the 49th International Scientific Conference on Information, Communication and Energy Systems and Technologies, ICEST 2014, Niš, Serbia, June 25 - 27, 2014, Vol.2, pp.347350, 2014.

[10] Z. Stankovic, N. Doncov, I. Milovanović, B. Milovanović, "Neural network model for efficient localization of a number of mutually arbitrary positioned stochastic EM sources in far-field", Proceedings of the 12th Symposium on Neural Network Applications in Electrical Engineering, NEUREL 2014, Beograd, Serbia, pp. 41-44, 2014. 
[11] Z. Stankovic, I. Milovanovic, N. Doncov, B. Milovanovic, „2D Localization of Source of Stochastic EM Radiation by using Neural Networks", LI International Scientific Conference on Information, Communication, and Energy Systems and Technologies - ICEST 2016 - Proceedings of Papers, pp. 99-102, 2016, ISBN: 978-9989-786-78-5.

[12] J.A. Russer, T. Asenov and P. Russer, "Sampling of stochastic electromagnetic fields", IEEE MTT-S International Microwave Symposium Digest, Montreal, Canada, pp. 1-3, 2012.
[13] J.A. Russer, P. Russer, "Modeling of Noisy EM Field Propagation Using Correlation Information", IEEE Transactions on Microwave Theory and Techniques, Volume 63, Issue 1, pp.76-89, 2015 